Meta

Journal des traducteurs

Translators' Journal

\title{
De la théorie à la pratique de la traduction
}

\section{Antin Fougner Rydning}

Volume 43, numéro 3, septembre 1998

URI : https://id.erudit.org/iderudit/004012ar

DOI : https://doi.org/10.7202/004012ar

Aller au sommaire du numéro

\section{Éditeur(s)}

Les Presses de l'Université de Montréal

ISSN

0026-0452 (imprimé)

1492-1421 (numérique)

Découvrir la revue

Citer cet article

Rydning, A. F. (1998). De la théorie à la pratique de la traduction. Meta, 43(3), 349-363. https://doi.org/10.7202/004012ar

\section{Résumé de l'article}

Cette étude a pour objectif la mise en application de la méthode interprétative de traduction à la traduction norvégienne d'un texte pragmatique français. Le cadre méthodologique dans lequel s'inscrit cette étude nous est fourni par L'analyse du discours comme méthode de traduction (Delisle 1980). La grande originalité de L'analyse résidant essentiellement au niveau du palier de l'exégèse lexicale, nous nous fonderons intégralement sur ce palier pour expliquer la création des équivalences contextuelles dans notre traduction. Les paliers de l'interprétation de la charge stylistique et de l'organicité textuelle étant traités de façon plus schématique, nous étofferons le modèle de Delisle en y insérant nos propres composantes ainsi que celles d'autres théoriciens du langage.
Ce document est protégé par la loi sur le droit d'auteur. L'utilisation des services d'Érudit (y compris la reproduction) est assujettie à sa politique d'utilisation que vous pouvez consulter en ligne.

https://apropos.erudit.org/fr/usagers/politique-dutilisation/ 


\title{
DE LA THÉORIE À LA PRATIQUE DE LA TRADUCTION
}

\author{
ANTIN FOUGNER RYDNING \\ Université d'Oslo, Oslo, Norvège
}

\begin{abstract}
Résumé
Cette étude a pour objectif la mise en application de la méthode interprétative de traduction à la traduction norvégienne d'un texte pragmatique français. Le cadre méthodologique dans lequel s'inscrit cette étude nous est fourni par L'analyse du discours comme méthode de traduction (Delisle 1980). La grande originalité de L'analyse résidant essentiellement au niveau du palier de l'exégèse lexicale, nous nous fonderons intégralement sur ce palier pour expliquer la création des équivalences contextuelles dans notre traduction. Les paliers de l'interprétation de la charge stylistique et de l'organicité textuelle étant traités de façon plus schématique, nous étofferons le modèle de Delisle en y insérant nos propres composantes ainsi que celles d'autres théoriciens du langage.
\end{abstract}

\begin{abstract}
The interpretive method as advocated by Delisle (1986) in L'analyse du discours comme méthode de traduction is applied to the translation from French to Norwegian of a pragmatic text. Our approach draws heavily on lexical exegesis for dealing with contextual equivalents. For interpreting stylistic features and addressing textual organicity, we have supplemented Delisle's method with a few ideas of our own and those of other translation theorists.
\end{abstract}

\section{INTRODUCTION}

Formulée par Jean Delisle en 1980, la méthode d'enseignement de la traduction dans L'analyse du discours comme méthode de traduction connaît un succès immédiat. Selon les modestes propos de son auteur, l'ouvrage a pour objet «la présentation d'une méthode originale d'initiation à la traduction française de textes pragmatiques anglais». La méthode est en fait bien plus qu'une méthode d'initiation à la traduction. Faisant ressortir les traits caractéristiques du processus cognitif de l'opération traduisante, elle mérite l'appellation de méthode de traduction tout court. Par ailleurs, bien que la méthode soit réservée à la traduction française de textes pragmatiques anglais, les principes fondamentaux dégagés ont une portée générale, universelle. Ils sont de ce fait applicables à toute traduction, quelles que soient les paires de langues mises en contact.

La méthode de traduction est originale en ce sens qu'elle ne se réduit pas à une simple présentation de cas particuliers ayant reçu une solution ad hoc. Prenant comme point de départ que la traduction est «une opération de jugement et de coordination qui consiste à concilier les impératifs sémantiques et stylistiques d'un discours tout en respectant les contraintes imposées par les règles d'écriture et l'organicité textuelle» (1980:124), la méthode est axée sur le transfert interlinguistique du sens du message. Rappelons le postulat à la base de la méthode : Le traducteur n'opère pas sur la langue, mais sur son emploi. Il s'agit pour le traducteur de comprendre le sens et de restituer celui-ci conformément à la stylistique de la langue d'arrivée.

Meta, XLIII, 3, 1998 
Notre propos est de mettre la méthode de traduction interprétative à l'épreuve d'une traduction norvégienne. Nous souhaitons montrer que la méthode vaut tout aussi bien pour la traduction qui met en présence la paire de langues français-norvégien, et ce faisant démontrer que la qualité de la traduction est directement liée à la faculté du traducteur de créer des équivalences contextuelles dans sa langue maternelle. Une traduction réussie est selon notre définition une traduction à la fois intelligible et constante dans la restitution du sens et des effets de sens de l'auteur. L'évaluation de l'intelligibilité du texte traduit s'effectuant sur les mêmes bases que celles qui valent pour l'acte de la communication unilingue, nous laisserons au lecteur le soin de juger de la conformité à la démarche de la langue d'arrivée. Mais afin de lui permettre d'entrevoir les mouvements complexes de la gymnastique mentale qui auront précédé les solutions retenues et guidé nos choix, nous accompagnerons notre traduction de commentaires.

La première partie de cette étude est consacrée à la présentation et à l'analyse stylistico-articulatoire du texte original français retenu pour notre traduction norvégienne. Les paliers de la charge stylistique et de l'organicité textuelle étant traités de façon relativement succincte par Delisle, nous avons été contrainte de nous appuyer sur d'autres ouvrages théoriques pour conduire cette partie de l'analyse. Nous nous sommes essentiellement fondée sur notre propre recherche (Rydning 1991) ainsi que sur les ouvrages de Vinay et Darbelnet (1977), Lundquist (1983) et Reboul (1984). Nous tenons cependant à préciser que nous n'avons pas pour autant dérogé au cadre méthodologique de Delisle, celui-ci restant parfaitement opératoire pour notre analyse. Le texte pragmatique autour duquel s'articule cette étude a été choisi essentiellement en fonction des trois critères suivants : sa brièveté, ses qualités stylistiques et son aptitude à brosser le tableau d'un problème d'actualité complexe. Nous déterminerons dans un premier temps la nature et la fonction du texte $(\S 1)$ avant de procéder à son analyse stylistique $(\S 2)$ et articulatoire $(\S 3)$.

La seconde partie abordera essentiellement les modalités de réalisation de l'opération traduisante. Après avoir proposé notre traduction norvégienne du texte $(\S 4)$, seront exposées dans une analyse justificative les difficultés rencontrées et les solutions retenues $(\S 5)$.

\section{LA NATURE DU TEXTE ET SA FONCTION}

Texte original français extrait du Monde, le 3 janvier 1994

\section{La maternité mécanisée}

Le désir d'enfant autorise-t-il à aller plus avant dans la médicalisation et l'instrumentalisation de la procréation?

Si l'on en croit Philippe Douste-Blazy, ministre délégué à la santé, le gouvernement français entend faire voter prochainement un texte de loi interdisant de manière explicite aux femmes ménopausées de donner naissance à un enfant. Plus précisément, les techniques de procréation médicalement assistée seraient réservées, selon le ministre, aux femmes «en âge de procréer, c'est-à-dire avant la ménopause».

Il s'agit là du dernier rebondissement hexagonal de l'intense polémique que provoque, depuis plusieurs années déjà, le recours aux techniques de fécondation in vitro en dehors du cadre thérapeutique de la lutte contre la stérilité. Soucieux de s'exprimer au plus vite dans ce domaine, le ministre délégué à la santé vient à sa manière alimenter cette polémique et obscurcir un peu plus les données d'un problème où la médecine et la science, totalement dépassées par ce qu'elles ont ici engendré, pas plus que la loi, ne semblent en mesure de fournir de réponses cohérentes.

Beaucoup plus prudent, le professeur Jean Bernard, président honoraire du Comité national d'éthique, expliquait il y a quelques jours, tout en condamnant cette pratique, que la loi semblait ici inopportune. Pour sa part, Simone Veil, ministre des affaires sociales et de la 
santé, vient de rappeler les termes du texte que l'Assemblée nationale a déjà adopté en première lecture sur ce thème.

La première et principale réponse instrumentale à la stérilité fut l'insémination artificielle avec le sperme de donneur, procédé qui permet de fournir une réponse thérapeutique à la plupart des stérilités d'origine masculine. Tout s'est brutalement compliqué avec la découverte, à la fin des années 70, que l'on pouvait, sans grande difficulté technique, obtenir la fécondation d'un ovule prélevé chez une femme par un spermatozoïde masculin en dehors des voies génitales féminines.

Cette fécondation in vitro, associée à la possibilité de conserver par congélation les cellules sexuelles et les embryons ainsi obtenus, multipliait à l'infini les possibilités de procréation et les «échanges» entre couples fertiles et stériles.

Dissociant de manière radicale la procréation de la relation sexuelle, ces techniques s'inscrivent dans le droit mouvement amorcé avec la mise au point des techniques contraceptives. Élargissent-elles pour autant le champ de liberté de l'espèce humaine ? Maîtriser de la sorte la fonction reproductive constitue-t-il un indispensable progrès ? Ces questions ne se posaient pas quand la procréation médicalement assistée n'était qu'une réponse à des situations pathologiques relativement communes et quand elle a été proposée à des couples considérés comme en âge de procréer. Leur stérilité était alors considérée comme une maladie. La biologie et la médecine ne faisaient qu'apporter un remède.

Les premières questions troublantes n'ont pas tardé. On vit ainsi des femmes seules ou homosexuelles réclamer la possibilité de «faire» un enfant grâce à l'insémination artificielle et des veuves demander le sperme, conservé par congélation, de leur défunt. Dans différents pays, des grands-mères assurèrent la gestation d'embryons obtenus après fécondation d'ovules de leur fille par des spermatozoïdes de leur gendre pendant que se multipliaient les dons de gamètes et d'embryons.

En France, la très rapide diffusion de ces techniques n'inquiéta pas les pouvoirs publics, qui ne prirent aucune mesure après l'échec de réglementation mise en place en 1987 par Michèle Barzach, alors ministre de la santé. Pour autant, un consensus devait se dégager chez les professionnels pour que la procréation médicalement assistée ne sorte pas du champ de la thérapeutique.

C'est donc à partir de l'étanger, d'Italie et de Grande-Bretagne en particulier, que le débat sur la légitimité et les limites de ces pratiques refait surface en France. S'interroger sur le fait de savoir si une femme a le droit ou non de donner naissance à un enfant après sa ménopause ne peut conduire à une réponse simple, ne serait-ce que parce que la ménopause ne renvoie pas à un âge strictement défini. Faudrait-il ainsi interdire aux femmes qui souffrent, pour des raisons médicales ou chirurgicales, de ménopause précoce d'avoir recours au don d'embryon ? Comment, d'autre part, le ministre délégué à la santé peut-il évoquer les risques de malformation des enfants ainsi conçus en fonction de l'âge de la femme en gestation, alors même que ces embryons sont issus d'ovules provenant de femmes qui sont en âge de procréer et pour lequel le risque de malformation est notablement inférieur?

«En France, les procréations médicalement assistées continuent de susciter l'intérêt passionné du public et chaque anecdote relatée par la presse amène son lot de propositions de réglementation, écrivait Noëlle Lenoir en 1991 dans son rapport sur la bioéthique. Mais la prudence a jusqu'ici prédominé sur l'action législative. Il est vrai que la décision de recourir aux procréations médicalement assistées, comme celle de faire don de gamètes ou d'un embryon, semblent relever au premier chef de la conscience des individus et des couples plus que la volonté de l'État.» En irait-il différemment avec les maternités post-ménopausiques ? Le principal argument invoqué par les opposants à cette pratique concerne l'intérêt de l'enfant à venir, le fait qu'il grandira auprès d'une femme âgée et qu'il sera, selon toute vraisemblance, tôt orphelin. Y a-t-il nécessité de légiférer ou faut-il s'en remettre au bon sens général et à la déontologie médicale ? L'erreur serait ici d'en rester à un problème certes spectaculaire, alimentant de nombreux fantasmes, mais en définitive relativement marginal.

Les vrais enjeux que soulèvent la diffusion des procréations médicalement assistée et l'instrumentalisation de la reproduction sont d'un autre ordre. Ils doivent au plus vite être analysés à la lumière de la demande grandissante de l'enfant «parfait» et de l'extraordinaire 
développement des techniques du diagnostic prénatal. Les nouvelles possibilités d'eugénisme ainsi offertes, grâce notamment à la nouvelle technique du diagnostic préimplantatoire, mériteraient, plus que les femmes ménopausées et leur désir tardif de grossesse, l'attention du ministre délégué à la santé, du gouvernement et des parlementaires.

\section{JEAN-YVES NAU}

L'article intitulé «La maternité mécanisée» est extrait de la première page du Monde du 3 janvier 1994. Le Monde est un quotidien d'informations générales considéré comme étant le journal de référence français par excellence. Il s'adresse au grand public cultivé s'intéressant à l'actualité. La mission de l'article de Jean-Yves Nau est d'informer les lecteurs du Monde des remous causés en France par l'initiative gouvernementale visant à faire voter au Sénat un projet de loi sur la bioéthique. S'adressant à un public informé mais non spécialiste en matière de bioéthique, l'article peut être défini comme un texte de vulgarisation. Le journaliste vise à expliquer à ses lecteurs les données du projet de loi qui interdit aux femmes ménopausées le recours aux techniques de procréation médicalement assistée.

Il s'agit d'un article de fond où le journaliste situe le projet de loi dans son contexte. Après l'échec de la tentative de réglementation du ministre de la Santé en 1987, il aura fallu attendre cinq ans (novembre 1992) pour faire adopter en première lecture par l'Assemblée nationale un texte de loi sur la procréation artificielle. Le journaliste rappelle qu'en soumettant ce texte à l'examen du Sénat en seconde lecture, le gouvernement ravive une intense polémique sur la légitimité et les limites des techniques de fécondation in vitro. La fonction du texte est essentiellement informative. Le journaliste s'efforce de relater les faits et les données du débat d'une façon objective. La première page de l'article est consacrée à l'exposition chronologique des diverses techniques de procréation médicalement assistée venues en réponse à la stérilité ainsi qu'aux problèmes inattendus qu'elles ont engendrés. Ce n'est que vers la fin du texte, soit la seconde page, que le journaliste «annonce ses couleurs» et profile son parti pris. La critique qu'il adresse aux pouvoirs publics devient virulente comme en témoignent les passages suivants :

Comment, d'autre part, le ministre délégué à la santé peut-il évoquer les risques de malformation des enfants ainsi conçus en fonction de l'âge de la femme en gestation, [...] ?

Y a-t-il nécessité de légiférer [...] ?

Les nouvelles possibilités d'eugénisme ainsi offertes, [...] mériteraient, plus que les femmes ménopausées et leur désir tardif de grossesse, l'attention du ministre délégué à la santé, du gouvernement et des parlementaires.

Au lieu de s'intéresser aux vrais enjeux soulevés par l'instrumentalisation de la procréation, tels ceux qui découlent du diagnostic préimplantatoire sur les embryons, le journaliste déplore que le gouvernement français se contente de réglementer des problèmes en définitive marginaux. Il souhaite visiblement convaincre ses lecteurs de la futilité de consacrer un texte de loi à des questions qui pourraient être résolues par le simple bon sens. À la fonction informative du texte se superpose ainsi une fonction appellative. L'objectif de l'article est double : informer le lecteur, mais aussi et surtout provoquer une réaction chez lui. Si ce dernier a pris la peine de lire la première page de son article, c'est qu'il souhaitait s'informer, se tenir au courant des données du débat avant de prendre parti pour ou contre la teneur du projet de loi. Ayant longtemps joué ses propres points de vue en mineur, le journaliste attend la fin de l'article pour faire connaître explicitement ses intentions et amener, au moyen du raisonnement, ses lecteurs à en reconnaître le bien-fondé. Une fois la lecture du texte terminée, le lecteur se rend compte que l'auteur l'a en fait subrepticement préparé, dès le début du texte, à prendre position en faveur de ses arguments.

Nul doute que les fonctions informative et appellative du texte devront être restituées dans une traduction qui vise à informer les lecteurs norvégiens du quotidien d'infor- 
mations générales Aftenposten du dernier rebondissement en France de la polémique concernant l'emploi des techniques de procréation en dehors de la lutte thérapeutique contre la stérilité. Il conviendra donc de recréer en norvégien un texte qui assume cette même double fonction. Nous rendrons compte ci-dessous d'un certain nombre des moyens stylistiques utilisés par l'auteur pour représenter ces fonctions informative et appellative.

\section{ANALYSE DE LA CHARGE STYLISTIQUE DU TEXTE}

L'article du Monde peut être conçu comme un texte informatif puisqu'il vise la présentation de faits relevant de la biotechnologie et du droit. Mais comme l'auteur émet également des jugements de valeur et vise l'adhésion des lecteurs à ses points de vue, une stratégie argumentative est mise en évidence par des actes de discours à la fois évaluatifs et persuasifs.

\subsection{Texte de vulgarisation}

De par son support, un quotidien d'informations générales, l'article s'adresse à des lecteurs qui ont des connaissances générales. Le journaliste, lui-même non spécialiste, vise à expliquer les données d'un problème à la fois médical et éthique. Il ne cherche pas à transmettre à ses lecteurs des connaissances spécialisées en matière de procréation médicalement assistée. Le niveau de langage de ce texte de vulgarisation est général, à quelques exceptions près. Pour s'assurer de la compréhension de ses lecteurs, l'auteur a recours aussi bien au choix d'unités terminologiques simples : femmes ménopausées / lutte contre la stérilité / fonction reproductive / techniques contraceptives qu'au procédé de l'expansion de l'information factuelle. Son langage est alors explicite, comme en témoignent d'une part les précisions apportées aux termes du projet de loi au moyen du connecteur explicatif (c'est nous qui soulignons) : [...] un texte de loi interdisant de manière explicite aux femmes ménopausées de donner naissance à un enfant. Plus précisément, les techniques de procréation médicalement assistée seraient réservées [...] aux femmes en âge de procréer [...] et, d'autre part, les définitions offertes de plusieurs notions clefs, définitions que l'on reconnaît au connecteur explicatif dans le premier exemple et au pronom démonstratif anaphorique dans le second (c'est nous qui soulignons) : femmes en âge de procréer, $c^{\prime}$ està-dire avant la ménopause / fécondation d'un ovule prélevé chez une femme par un spermatozö̈de masculin en dehors des voies génitales féminines. Cette fécondation in vitro [...]. Dans la seconde moitié du texte, le registre de langue devient légèrement plus spécialisé. Les unités terminologiques suivantes : déontologie médicale / eugénisme / technique du diagnostic pré-implantatoire ne sont pas accompagnées d'explication, ce qui laisse présumer que l'auteur s'attend à ce que leur sens découle du contexte cognitif.

\subsection{Stratégie argumentative}

La fonction appellative du texte est suggérée plutôt qu'exprimée de façon explicite. Se cache derrière une narration qui se veut objective des jugements subjectifs, lesquels permettent de classer le texte comme un acte de discours évaluatif. Mais en plus de relater et d'ordonner des événements, des comportements et des faits de façon à faire ressortir clairement ses opinions personnelles, l'auteur s'efforce de convaincre les lecteurs par ses raisonnements. Cet acte par lequel l'auteur cherche à amener ses lecteurs à prendre position est persuasif.

Le thème qui préoccupe le journaliste au premier chef est de savoir si le recours à une légifération pour déterminer les limites de la procréation médicalement assistée est réellement nécessaire. Bien que l'auteur n'intervienne pas directement dans le texte pour nous rapporter son attitude au moyen de tournures déclaratives, le lecteur comprend - 
derrière l'objectivité apparente des faits décrits - que l'auteur est critique. Un indice de cette attitude est le nombre d'expressions valorisées qui apparaissent au $\S 2$, telles alimenter cette polémique / obscurcir un peu plus les données d'un problème / ne semblent en mesure de fournir de réponses cohérentes. Cette impression de jugement critique, perçue en début de texte, est confirmée aux deux derniers paragraphes qui abondent en expressions chargées de valeur : Le principal argument invoqué par les opposants à cette pratique / L'erreur serait [...] d'en rester à un problème certes spectaculaire, alimentant de nombreux fantasmes, mais en définitive relativement marginal / Les vrais enjeux [...] sont d'un autre ordre. Ils doivent au plus vite être analysés / Les nouvelles possibilités d'eugénisme [...] mériteraient l'attention du ministre [...].

Un second procédé stylistique qui contribue à mettre en relief l'opinion personnelle du journaliste est l'emploi de l'argument d'autorité. L'auteur cite au $\S 3$ une personne considérée comme particulièrement compétente en la matière à l'appui de sa position, en l'occurrence le professeur Jean Bernard, président honoraire du Comité national d'éthique. L'argument d'autorité est cependant immédiatement opposé à la position de Mme Simone Veil. L'auteur parvient par ce moyen à concentrer l'objet de discorde.

Un troisième procédé stylistique qui sert à profiler le parti pris de l'auteur est celui de la prolepse, figure de pensée qui consiste à énoncer d'avance l'objection de ses adversaires, puis à la retourner contre eux. Le $\S 12$ s'ouvre sur les propos suivants : «Le principal argument invoqué par les opposants à cette pratique concerne l'intérêt de l'enfant à venir [...]». Plutôt que de réfuter cette objection, l'auteur en minimise fort subtilement l'impact en ces termes : «L'erreur serait ici d'en rester à un problème certes spectaculaire [...], mais en définitive relativement marginal.»

Mentionnons enfin un procédé auquel l'auteur a recours à plusieurs reprises pour faire ressortir son jugement, celui de l'interrogation rhétorique, dite aussi question oratoire. Les questions posées dans l'article ne nécessitant aucune réponse de la part du lecteur, elles peuvent de ce fait être considérées comme des questions fictives. La particularité de ce procédé stylistique est néanmoins d'amener le lecteur à raisonner tout en lui suggérant une réponse évidente - réponse qui coïncidera avec l'opinion de l'auteur... La première occurrence de l'interrogation rhétorique intervient dès le sous-titre. Elle a pour effet de confronter d'emblée le lecteur à un problème d'ordre éthique et philosophique important : celui de savoir si la fin justifie les moyens en matière de procréation artificielle. Les deux occurrences suivantes au $\S 6$ détaillent le problème soulevé. Mais, pas plus que la première, elle autorisent plus d'une seule réponse. La maîtrise de la fonction reproductive ne constitue aucune garantie de la liberté de l'espèce humaine, ni aucun progrès scientifique indispensable. Les occurrences de l'interrogation rhétorique aux $\S \S$ 10,11 et 12 servent essentiellement à mettre en relief le thème qui tient le journaliste particulièrement à cœur : la futilité de réserver un texte de loi à l'interdiction des techniques de procréation médicalement assistée aux femmes ménopausées. Comme pour les occurrences précédentes, la réponse à ces questions rhétoriques est à chaque fois non. La fonction de la question partielle posée au § 10 est différente. N'appelant pas de réponse, son

rôle est sinon de ridiculiser l'action gouvernementale, du moins d'exprimer des doutes sur les capacités de jugement du ministre délégué à la santé. Ce procédé stylistique qui a pour effet d'établir un contraste entre deux conceptions, offre au lecteur les moyens de prendre position.

\section{L'ORGANICITÉ DU TEXTE}

Le palier de l'organicité textuelle est celui de la dynamique du texte; il renvoie à «l'interdépendance hiérarchisée de tous les éléments d'un texte.» (Delisle 1980: 119). 
Parmi les procédés articulatoires qui contribuent à donner son élan au texte, nous mettrons l'accent sur quatre indices : la disposition matérielle du texte, les charnières du texte, les interrogations rhétoriques et l'hypotaxe.

\subsection{La disposition matérielle du texte}

La présence de titres et le découpage en 13 paragraphes sont les indices matériels les plus évidents de la structuration du texte. Le titre du texte «La maternité mécanisée»a pour effet d'orienter la lecture vers le spectaculaire. Il met en relief l'un des thèmes principaux du texte, celui des dangers de l'acharnement procréatique. Ce thème est repris dans le sous-titre - non moins spectaculaire - formé comme une question oratoire dont le but, avons-nous dit, est d'accrocher l'attention du lecteur et de le contraindre à réfléchir à la question de savoir s'il est légitime de considérer l'enfant comme un dû et, le cas échéant, s'il convient de poursuivre les recherches dans le domaine de la fécondation artificielle. L'introduction de ce thème sert de tremplin au jugement critique que réserve le journaliste au texte de loi. L'absence de pertinence de ce texte de loi est développée avec force tout le long du texte. À chaque paragraphe correspond soit une énumération de détails destinés à frapper le lecteur, soit un argument visant à emporter la conviction de celui-ci.

\subsection{Les charnières du texte}

Les charnières du texte servent à articuler des raisonnements, à faire avancer l'argumentation, et elles peuvent de ce fait être considérées comme des facteurs constitutifs de la cohérence thématique. Donnons-en quelques exemples. Au $\S 3$, l'opinion défavorable du professeur Bernard à l'égard de la position gouvernementale est aussitôt opposée à celle du ministre des Affaires sociales et de la Santé, Simone Veil. Le connecteur adversatif pour sa part sert à introduire la divergence qui existe entre deux attitudes mentales face à une même situation. Après une présentation des différentes techniques de procréation et de leurs conséquences, le $\S 8$ introduit l'idée centrale du texte, celle de savoir si à l'action législative peuvent être substituées la déontologie médicale et la conscience individuelle. Le connecteur pour autant, à valeur concessive, sert à postuler un lien entre le bon sens des médecins et l'échec de la tentative de réglementation en 1987. Ce procédé, par lequel l'auteur pose en quelque sorte les données du problème, peut ainsi être considéré comme un facteur d'agencement du texte. Les trois types de connecteurs au $\S 13$ : adversatif, temporel et conclusif servent quant à eux à préciser l'intention de l'auteur. Le premier, le connecteur adversatif d'un autre ordre, sert à mettre l'accent sur une idée concurrente à celle qui vient d'être exposée, à savoir la nécessité de procéder à une réflexion plus nuancée, plus poussée, plus complexe sur les vrais enjeux de la bioéthique. Le second, le connecteur temporel au plus vite souligne la nécessité d'offrir une réaction immédiate aux problèmes pressants, alors que le troisième, le connecteur conclusif ainsi sert à annoncer la fin d'un raisonnement. Tout en clôturant le débat, cette charnière de terminaison renforce la solution proposée : celle de réserver une réglementation aux problèmes soulevés par le diagnostic préimplantatoire touchant à l'eugénisme. Malgré leurs fonctions différentes, ces connecteurs servent à faire avancer l'argumentation et peuvent de ce fait être considérés comme des facteurs d'agencement du texte.

\subsection{L'interrogation rhétorique}

Le journaliste relate aux $\S \S 4,5$ et 6 l'évolution des techniques de procréation. Cette description est doublée, à partir de la fin du $\S 6$, d'interrogations rhétoriques. Nous avons commenté au $\S 2.2$. ci-dessus ces interrogations rhétoriques dans une optique stylistique. Mais comme elles contribuent à élargir le débat en lui fournissant une dimension éthique, les interrogations rhétoriques qui figurent aux $\$ \S 9-12$ peuvent également être considérées 
comme des indices de cohérence thématique. Elles peuvent de ce fait être analysées dans une optique articulatoire. Le sentiment d'absurdité évoqué par la question posée au $\S 9$ se trouve renforcé par l'interrogation fictive au $\S 10$, où est précisée l'attitude défavorable de l'auteur face à l'action gouvernementale. Ne pouvant appeler de réponse - car il s'agit bien d'une question partielle - cette interrogation sert néanmoins de réponse à la question posée au $\S 9$, et fonctionne ainsi comme facteur de cohérence thématique. Les deux occurrences de l'interrogation rhétorique aux $\S \S 11$ et 12 agissent dans le même sens. Elles nous permettent de comprendre le pourquoi de la réprobation du journaliste. Réserver un texte de loi à l'interdiction des techniques de procréation médicalement assistée aux femmes ménopausées lui semble un acte futile. Les réponses que donne l'auteur à ces questions au dernier paragraphe permettent d'accéder pleinement au sens du message du texte. C'est donc en partie grâce à ce procédé articulatoire que nous voyons la position du journaliste se concrétiser et se dresser en une violente critique des pouvoirs publics. Non seulement est-il d'avis qu'une action législative relative à un problème marginal devrait céder la place au bon sens, mais aussi est-il offusqué par l'absence d'initiative législative face aux véritables problèmes soulevés par la procréation médicalement assistée, à savoir ceux qui portent sur l'eugénisme.

\subsection{L'hypotaxe}

Nous ne relèverons ici qu'une seule caractéristique de la syntaxe plutôt complexe du texte, à savoir le recours fréquent à l'hypotaxe, procédé de subordination des propositions. Les nombreuses informations qui y sont condensées tout au long du texte sont subordonnées conformément à «la tendance du français à lier les faits par le raisonnement.» (Vinay et Darbelnet $1977: 229)$. Le passage ci-dessous est un exemple typique de ce procédé articulatoire qui joue un rôle dominant dans ce texte.

Dans différents pays, des grands-mères assurèrent la gestation d'embryons obtenus après fécondation d'ovules de leur fille par des spermatozoïdes de leur gendre pendant que se multipliaient les dons de gamètes et d'embryons.

La locution conjonctive pendant que a pour effet de relier deux propositions porteuses de deux idées différentes à l'intérieur d'un même champ sémantique : celui de la procréation. Sans pour autant créer un lien logique entre ces deux propositions, la locution conjonctive les articule l'une par rapport à l'autre et constitue de ce fait un facteur d'agencement du texte.

\section{TRADUCTION NORVÉGIENNE DE L'ARTICLE LA MATERNITÉ MÉCANISÉE}

La traduction norvégienne est destinée au quotidien norvégien Aftenposten. La mission de la traduction est d'informer les lecteurs norvégiens d'Aftenposten du débat sur les techniques de procréation médicalement assistée qui se déroule actuellement en France dans le sillage du texte de loi présenté par le gouvernement français pour adoption au Sénat.

\section{Barn på bestilling ?}

I hvor stor grad kan фnsket om à få barn rettferdiggjøre bruk av avansert reproduksjonsteknologi?

Ifølge visehelseminister Philippe Douste-Blazy, akter den franske regjering om kort tid å fremme et lovforslag for Senatet som uttrykkelig forbyr kvinner som har passert overgangsalderen å føde barn. I praksis vil kunstig befruktning, slik Douste-Blazy uttrykte det, bli forbeholdt «befruktningsdyktige kvinner, dvs. kvinner som ennå ikke har nådd klimakteriet». 
Lovforslaget er det siste utspillet i en årelang meningsstrid som har rast i Frankrike i saken om hvorvidt prøverørsmetoden skal tillates brukt $\mathrm{i}$ andre $\varnothing y e m e d$ enn ved behandling av befruktningsudyktighet. I sin iver etter å gi sitt syn til kjenne snarest mulig, bidrar DousteBlazy til å nøre opp under striden og til ytterligere å tåkelegge fakta. Fullstendig ute av stand til å kontrollere den utviklingen den selv har satt igang, synes legevitenskapen, like lite som lovverket, i stand til å komme med fornuftige svar på problemene.

Ærespresidenten i det franske etikkutvalget, professor Jean Bernard, var langt mer forsiktig i sine uttalelser. Til tross for at han fordømmer dagens praksis, ga han for noen dager siden uttrykk for at loven her syntes lite hensiksmessig. Helse- og sosialminister Simone Veil minnet for sin del nylig om ordlyden i det lovforslaget som Nasjonalforsamlingen vedtok ved første gangs behandling.

Det første og viktigste botemiddel mot ufrivillig barnløshet var kunstig inseminasjon med sæd fra giver. Denne metoden gjorde det mulig å behandle de fleste tilfeller av mannlig befruktningsudyktighet. Alt ble med ett langt vanskeligere da man på slutten av 70-tallet fant ut at befruktning enkelt kunne oppnås ved å forene en eggcelle med en sædcelle utenfor kvinnens kropp. Denne befruktningsteknikken (in vitro fertilisering) kombinert med teknikkene for å oppbevare kjønnsceller og befruktede egg ved nedfrysning, økte nærmest ubegrenset mulighetene for befruktning og «utveksling» mellom fruktbare og ufruktbare par. Disse teknikkene skiller forplantningen radikalt fra seksualakten og skriver seg således direkte inn i den prosessen som startet med utviklingen av prevensjonsmidler. Men bidrar de dermed til å $\varnothing \mathrm{ke}$ menneskets frihet? Er det faktum at vi er herre over vår egen forplantningsevne ensbetydende med fremskritt? Disse spørsmålene ble ikke stilt så lenge kunstig befruktning ble betraktet som en løsning på et relativt utbredt sykdomstilfelle og kun ble tilbudt par i befruktningsdyktig alder. Ufrivillig barnløshet ble den gang sett på som en sykdom, og bioteknologien bidro ganske enkelt til å helbrede denne sykdommen.

De første urovekkende spørsmålene lot ikke lenge vente på seg. Enslige eller homoseksuelle kvinner krevde retten til å få barn ved hjelp av kunstig inseminasjon, og enker ba om å bli befruktet med den avdøde ektefellens nedfrosne sæd. I flere land sørget bestemødre for å bære frem sine tilkommende barnebarn etter at datterens egg var blitt befruktet med sædceller fra svigersønnen. Parallelt steg antall donasjoner av kjønnsceller og befruktede egg.

Den svært raske spredningen av disse nye teknikkene bekymret ikke myndighetene nevneverdig. De kom ikke med et eneste tiltak etter at daværende helseminister Michèle Barzachs fors $\varnothing \mathrm{k}$ på å få istand en lovgivning strandet i 1987. Ikke desto mindre gikk legestanden inn for at tilbudet om kunstig befruktning skulle forbeholdes par med medisinske problemer.

Det er altså med utgangspunkt i erfaringer fra utlandet, og da især fra Italia og Storbritannia, at debatten rundt berettigelsen og begrensningen av kunstig befruktning på ny blusser opp i Frankrike. Spørsmålet om en kvinne skal ha lov til å føde barn etter klimakteriet kan ikke besvares med et enkelt ja eller nei, all den tid klimakteriet ikke kan avgrenses til en bestemt alder. Skulle man i så fall nekte kvinner, som av medisinske eller kirugiske årsaker lider av fremskutt overgangsalder, muligheten til å bli kunstig befruktet ?

Av Jean-Yves Nau

\section{L'ANALYSE JUSTIFICATIVE : DIFFICULTÉS TRADUCTIONNELLES ET SOLUTIONS}

Le processus traductionnel se réalise en trois temps. Il comprend une phase de compréhension du vouloir-dire de l'auteur, une phase de reformulation du sens compris et une phase de justification. C'est dans le cadre de cette troisième phase qui a pour but de vérifier que les choix effectués rendent bien compte des idées contenues dans le texte original en vue d'une mise en forme définitive de la traduction, que nous évoquerons les difficultés auxquelles nous nous sommes heurtée au cours de la traduction et que nous rendrons compte des solutions apportées aux problèmes. Pour permettre aux lecteurs qui ne maîtrisent pas le norvégien de suivre les contours de notre raisonnement, nous avons muni les passages commentés de notre traduction norvégienne d'un transcodage français (indiqué 
par la mention TF). Par le biais du français, nous visons donc la transparence des solutions syntaxico-sémantiques que nous avons retenues dans notre traduction norvégienne. Mais pour conférer aux passages transcodés un minimum d'intelligibilité, nous avons veillé à assurer leur grammaticalité.

\subsection{Les problèmes de compréhension}

Pour pouvoir traduire, il faut au préalable avoir compris le texte original. La compréhension est une condition sine qua non en traduction, car on ne peut reformuler intelligiblement que ce qu'on a réellement compris.

Nous avons vu que le vocabulaire se composait d'un certain nombre de termes spécialisés qui, tant qu'ils ne sont pas maîtrisés, contribuent à bloquer la compréhension. Il s'agit d'une part de termes qui relèvent de la médecine, tels :

- procréation médicalement assistée;

- gestation d'embryons;

- ménopause précoce;

- gamètes;

- déontologie médicale;

- eugénisme.

Il s'agit d'autre part de termes qui relèvent du droit tels :

- texte de loi;

- première lecture;

- tentative de réglementation;

- légitimité et limites de ces pratiques;

- action législative;

- nécessité de légiférer.

Dans la plupart des cas, le recours au dictionnaire unilingue (Petit Larousse) aura permis de comprendre la signification de ces termes.

La complexité de certaines tournures syntaxiques risque également de bloquer l'appréhension du sens. Donnons comme exemple les deux propositions suivantes extraites respectivement des $\S 2$ et 7 :

$\S 2[\ldots]$ le ministre délégué à la santé vient à sa manière alimenter cette polémique et obscurcir un peu plus les données d'un problème où la médecine et la science, totalement dépassées par ce qu'elles ont ici engendré, pas plus que la loi, ne semblent en mesure de fournir de réponses cohérentes.

Nous reconnaissons ici une tendance vers le développement raisonné. L'auteur retarde en quelque sorte le «déroulement des idées jusqu'à ce qu'il les ait ordonnées, qu'il en ait dégagé la succession, l'ordonnance cachée, la cause et l'effet» (Vinay et Darbelnet 1977 : 221). Il se place sur le plan de l'entendement. Une décomposition en fonction de leur émergence chronologique permet de retenir les trois idées imbriquées dans cette structure. Celles-ci peuvent être paraphrasées comme suit :

1) Les progrès récents dans le domaine de la procréation artificielle soulèvent de nombreux problèmes qui préoccupent vivement les Français.

2) Le projet de loi de Douste-Blazy ne contribue aucunement à résoudre ces problèmes dûs aux expérimentations, applications et manipulations nouvelles jusque-là inimaginées par la médecine. 
3) Ces types d'expérimentations devraient être soumis à un contrôle législatif, mais il n'existe à l'heure actuelle aucune réglementation adéquate.

Il en va de même pour :

§ 7 Dans différents pays, des grands-mères assurèrent la gestation d'embryons obtenus après fécondation d'ovules de leur fille par des spermatozoïdes de leur gendre pendant que se multipliaient les dons de gamètes et d'embryons.

Les rapports entre la grand-mère, sa fille, son gendre et leur embryon présentés dans la principale sont reliés à une proposition temporelle sur la multiplication des dons de gamètes et d'embryons. Cette façon de relier entre elles deux idées qui n'ont aucun lien logique autre que le fait d'être des coutumes pratiquées à l'étranger risque de dérouter le lecteur norvégien, davantage habitué à une subdivision marquée par la ponctuation pour distinguer une idée d'une autre. Pour voir comment nous avons choisi de traduire ces deux énoncés, nous renvoyons au point 5.2. ci-après, où sont exposées nos solutions aux problèmes.

\subsection{Solutions de reformulation}

Philippe Douste-Blazy est ministre délégué à la santé. Son supérieur hiérarchique est Simone Veil, ministre d'État des Affaires sociales, de la Santé et de la Ville. Le problème est ici de savoir quel terme choisir dans la traduction norvégienne pour restituer la différence entre le ministre d'État et le ministre délégué - le partage des fonctions ne s'effectuant pas de la même façon en Norvège. Après avoir consulté l'ancien ministre de la Culture, M. Roald Langslet, nous avons opté pour visehelseminister (TF : vice-ministre de la santé). Puisque les deux ministres étaient mentionnés dans l'article, il nous fallait obligatoirement choisir un terme susceptible de marquer la distinction entre eux. Dans une situation de communication différente, admettons un texte qui ne fasse aucune allusion à Simone Veil, il nous aurait suffi de désigner M. Douste-Blazy au moyen de : helseministeren ( $\mathrm{TF}$ : ministre de la Santé). Notons que le nom propre en français est antéposé par rapport au titre, alors qu'il est au contraire postposé en norvégien :

Ifølge vise-helseminister Philippe Douste-Blazy...

TF :Selon le vice-ministre de la santé Philippe Douste-Blazy...

Notre second commentaire porte sur la traduction du passage suivant :

le gouvernement français entend faire voter prochainement un texte de loi [...]

Plutôt que de recourir au transcodage suivant :

den franske regjering akter om kort tid å få vedtatt et lovforslag [...]

nous avons choisi la solution ci-dessous légèrement plus étoffée par rapport à l'original :

den franske regjering akter om kort tid å legge frem et lovforslag for Senatet [...]

$\mathrm{TF}$ :le gouvernement français compte présenter prochainement au Sénat un texte de loi [...]

Il nous a en effet semblé pertinent de fournir à nos lecteurs le complément d'information nécessaire pour comprendre l'idée véhiculée par l'auteur de la même façon que l'ont comprise les lecteurs français. Le contexte cognitif, en l'occurrence les informations qui nous sont fournies au $\S 3$ sur l'adoption du texte de loi en première lecture par l'Assemblée nationale, permet aux lecteurs français de comprendre d'emblée que le texte de loi doit être voté en seconde lecture par le Sénat pour être définitivement adopté. L'auteur a choisi de supposer connues chez ses lecteurs français les connaissances pertinentes en 
matière d'adoption d'un texte législatif. Estimant que les lecteurs norvégiens ont besoin d'un complément d'information pour comprendre la même chose que les lecteurs français, nous avons choisi le procédé qui consiste à expliciter une information, qui dans le texte original, est implicite. Le sens est néanmoins intact. Nous avons délibérément opté pour une légère explicitation plutôt qu'une explicitation plus prononcée, telle l'emploi d'une parenthèse où nous ajoutons que le texte de loi a déjà été voté en première lecture par l'Assemblée nationale. Nous estimons en effet que le contexte cognitif permet aux lecteurs norvégiens d'établir eux-mêmes le rapport entre la première lecture par l'Assemblée nationale et la seconde lecture par le Sénat, rapport que les lecteurs français doivent eux aussi obligatoirement établir.

$\mathrm{Au} \S 2$ intervient l'adjectif hexagonal qui en norvégien correspond à sekskantet. Ce terme renvoie à la France métropolitaine, pays qui de par sa forme ressemble à un hexagone. Le terme d'hexagone étant couramment employé comme synonyme de la France, il suffit de le traduire par Frankrike sans donner de précisions supplémentaires quant à ses six côtés. Comme l'accent n'est pas mis sur la forme de la France dans ce texte, il est superflu d'actualiser cet aspect dans la traduction. Rappelons que le but d'une traduction est de véhiculer le sens et non les significations potentielles des mots du texte.

Le terme médical fécondation in vitro a été rendu en norvégien par in vitro fertilisering. Cette correspondance de termes est à considérer comme un report de vocables monosémiques, c'est-à-dire de vocables à signification unique. Il s'agit, pour reprendre les termes de Delisle, «des éléments d'information qui échappent presque complètement à l'analyse exégétique, au raisonnement analogique» (1980: 101). Ces vocables peuvent être reportés tels quels dans la langue d'arrivée. Si nous avons choisi ce procédé de transcodage, c'est parce que nous avons opté pour la même ordonnance des éléments de sens que l'auteur: la définition de cette unité terminologique étant offerte deux paragraphes plus loin dans le texte original, nous avons estimé que le lecteur norvégien qui en ignorerait la signification pouvait, tout comme le lecteur ignorant le français, s'inspirer du contexte cognitif pour comprendre.

Nous avons en revanche choisi de rendre le terme embryon, non pas par le terme correspondant embryo en norvégien, mais par le terme befruktet egg (TF : ovule fécondé). Pourquoi une solution d'extension dans ce cas particulier alors que celle-ci même avait été rejetée pour le cas de fécondation in vitro ? La solution consistant à traduire par befruktet egg a été dictée par d'autres impératifs. Le terme embryon n'étant pas défini dans le texte, contrairement à fécondation in vitro, c'est le souci de transparence, de compréhension immédiate des lecteurs non spécialistes en matière de fécondation qui a imposé ce choix. En dépit des apparences, le terme embryo en norvégien relève d'un registre de langue plus specialisé que son correspondant embryon en français. Tout Français comprend d'emblée la signification du terme, alors que tout Norvégien ne comprend pas nécessairement la signification de embryo. Et puisque nous adressons notre traduction non pas à des spécialistes en biotechnologie, mais aux lecteurs d'Aftenposten, il nous a paru important de leur en faciliter la lecture là où cela était possible, sans pour autant trahir le style assez dense du texte.

Revenons ici aux deux constructions problématiques que nous avions signalées au point 5.1. ci-dessus. La première :

Soucieux de s'exprimer au plus vite dans ce domaine, le ministre délégué à la santé vient à sa manière alimenter cette polémique et obscurcir un peu plus les données d'un problème où la médecine et la science, totalement dépassées par ce qu'elles ont ici engendré, pas plus que la loi, ne semblent en mesure de fournir de réponses cohérentes.

Nous l'avons traduite en norvégien en ces termes : 
I sin iver etter å gi sitt syn til kjenne snarest mulig i denne saken, bidrar visehelseministeren til å nore opp under striden og til ytterligere å tåkelegge fakta. Fullstendig ute av stand til å kontrollere den utviklingen den selv har satt igang, synes legevitenskapen, like lite som lovverket, $i$ stand til å komme med fornuftige svar på problemene.

$\mathrm{TF}$ :Empressé de faire connaître son point de vue le plus vite possible dans cette affaire, le vice-ministre de la Santé contribue à attiser le conflit et à obscurcir davantage les faits. Dans l'incapacité absolue de contrôler le développement qu'elle a elle-même engendré, la science médicale, pas plus que la loi, ne semble être en mesure de fournir de réponses raisonnables aux problèmes.

Nous avons opté pour un développement beaucoup plus sensoriel en norvégien en faisant correspondre à la longue phrase française deux phrases en norvégien. Leur enchaînement a été fait en fonction d'un ordre chronologique : les idées sont présentées au fur et à mesure de leur émergence. Cette façon de présenter les événements dans leur déroulement temporel est, à notre avis, conforme au style narratif du norvégien. Nous avons également choisi de juxtaposer ces deux phrases du fait que ce procédé nous semble convenir à la démarche usuelle du norvégien, à son comportement logique. Mais puisque la seconde phrase est porteuse de deux idées, nous avons tenu à agencer celles-ci au moyen de la charnière explicite like lite. Nous remarquons a posteriori que ce syntagme a été transcodé, l'auteur ayant opté pour pas plus que dans son texte.

Notons aussi que nous avons choisi de réunir les deux mentions médecine et science sous le terme générique de legevitenskap (TF : science médicale) en norvégien. À notre avis, le procédé de l'implicitation nous semble plus approprié pour restituer l'idée que ce sont l'ensemble des connaissances scientifiques médicales qui sont actualisées par le contexte.

Quant au deuxième énoncé problématique déjà signalé au point 5.1. ci-dessus :

Dans différents pays, des grands-mères assurèrent la gestation d'embryons obtenus après fécondation d'ovules de leur fille par des spermatozoïdes de leur gendre pendant que se multipliaient les dons de gamètes et d'embryons

nous avons, ici aussi, choisi d'agencer les faits autrement qu'en français :

I forskjellige land sфrget bestem $\phi$ dre for å bare frem sine tilkommende barnebarn etter at døtrenes egg var blitt befruktet med sadceller fra svogeren. Parallelt steg antall donasjoner av kjønnsceller og befruktede egg.

$\mathrm{TF}$ :Dans différents pays, des grands-mères veillèrent à porter leurs futurs petits-enfants après que les ovules de leurs filles furent fécondées par des spermatozoïdes du gendre. Parallèlement, le nombre de dons de gamètes et d'ovules fécondés augmentait.

Les deux idées sont exposées dans deux phrases différentes mais reliées l'une à l'autre au moyen du connecteur additif parallelt. Ce choix nous a été dicté par la démarche plus usuelle du développement sensoriel en norvégien.

Concluons ce chapitre par l'analyse de deux problèmes syntaxiques. Le premier porte sur la polysémie créée par le gérondif dans la phrase suivante au $\S 3$ :

Beaucoup plus prudent, le professeur Jean Bernard, président honoraire du Comité national d'éthique, expliquait il y a quelques jours, tout en condamnant cette pratique, que la loi semblait ici inopportune.

Le gérondif renforcé par tout peut exprimer tant la simultanéité que la concession. Vu que le norvégien n'est pas doté d'une construction syntaxique semblable susceptible de rendre ces deux significations, il va de soi que le traducteur est obligé de choisir. Ce choix ne sera cependant pas fortuit, puisque rien ne porte à croire que l'auteur a sciemment cherché l'ambiguïté. Le contexte cognitif nous fournit la solution au problème de l'interprétation probable du vouloir-dire de l'auteur. Est mis en relief par cette construction un 
paradoxe qui consiste à réfuter un projet de loi visant à réglementer tout recours aux techniques de procréation médicalement assistée en dehors du cadre thérapeutique de la lutte contre la stérilité, alors qu'est condamnée la pratique de ces techniques en dehors du champ thérapeutique... La reformulation en norvégien ne pose dès lors aucun problème. C'est bien l'idée de la concession qu'il faudra veiller à restituer. Une solution possible est de recourir en norvégien au connecteur concessif til tross :

Erespresidenten $i$ det franske etikkutvalget, professor Jean Bernard, var langt mer forsiktig $i$ sine uttalelser. Til tross for at han fordommer dagens praksis, ga han for noen dager siden uttrykk for at loven her syntes lite hensiktsmessig.

$\mathrm{TF}$ :Le président honoraire du Comité français d'éthique, le professeur Jean Bernard, était beaucoup plus prudent dans ses déclarations. Bien qu'il condamne la pratique actuelle, il expliquait il y a quelques jours que la loi semblait ici inappropriée.

Notre dernier commentaire porte sur la phrase suivante, extraite du $\S 8$ :

Pour autant, un consensus devait se dégager chez les professionnels pour que la procréation médicalement assistée ne sorte pas du champ de la thérapeutique.

La construction devait + infinitif exprime une action qui s'est effectivement produite dans le passé. Elle sert à souligner que le futur du passé a été réalisé. En norvégien, la restitution de cette idée de fait accompli dans le passé s'effectue au moyen d'une tournure au passé :

Ikke desto mindre ble ekspertene enige om at kunstig befruktning bare skulle forbeholdes behandling av sterilitet.

TF :Pour autant, les experts convinrent que la fécondation artificielle devait uniquement être réservée au traitement de la stérilité.

Ajoutons enfin, au sujet de la solution retenue, que nous avons choisi de traduire l'idée plutôt abstraite contenue dans la complétive par la tournure beaucoup plus concrète : [...] at kunstig befruktning bare skulle forbeholdes behandling av sterilitet (TF : que la fécondation artificielle devait uniquement être réservée au traitement de la stérilité). Cette équivalence phrastique contextuelle nous semble particulièrement appropriée pour restituer le sens exprimé. Une traduction basée sur la correspondance des termes : [...] at kunstig befruktning ikke skulle gå utover det terapeutiske nous paraît bien plus imprécise et nettement moins conforme à la démarche du norvégien. Cet exemple d'équivalence correspond à ce que Delisle nomme la recréation contextuelle. Il s'agit d'une équivalence idéique qui n'est pas consignée dans la langue d'arrivée. Elle résulte d'une analyse exégétique du texte.

\section{CONCLUSION}

Nous fondant sur la théorie interprétative de l'école de Paris, nous avons mis en application la méthode de traduction interprétative dans notre traduction norvégienne de l'article intitulé «La maternité mécanisée» de Jean-Yves Nau. Nous avons accompagné nos solutions de commentaires dans l'espoir de montrer que l'opération traductionnelle est un processus cognitif dynamique requérant de la part du traducteur une part considérable d'observation, d'intuition, de jugement et d'intelligence.

Les problèmes soulevés dans cette étude ainsi que les solutions retenues auront, espérons-le, servi à réfuter l'idée commune selon laquelle la traduction se ramènerait à une simple recherche statique de correspondances assignées dans les deux systèmes linguistiques. Le traducteur, à cheval entre deux systèmes linguistiques, deux cultures, deux publics différents, est — tout au long de son travail — amené à faire de nombreux choix. Il 
effectue ceux-ci non pas uniquement en fonction des deux langues en contact, mais en fonction d'une multitude de facteurs communicatifs. Nos commentaires, pour l'essentiel axés sur les modalités de réalisation de l'opération traduisante, auront permis de mettre l'éclairage sur certains d'entre eux.

\section{RÉFÉRENCES}

BALLARD, Michel (dir.) (1984) : La traduction - De la théorie à la didactique, Lille, Université de Lille III.

DELISLE, Jean (1980) : L'analyse du discours comme méthode de traduction, Ottawa, Édition de l'Université d'Ottawa.

LUNDQUIST, Lita (1983) : L'analyse textuelle, Paris, Cedic.

PEDERSEN, John, SPANG-HANSSEN, Ebbe \& Carl VIKNER (1980) : Fransk Grammatikk, Copenhague, Akademisk forlag.

REBOUL, Olivier (1984) : La rhétorique, Paris, Presses Universitaires de France.

REY-DEBOVE, Josette et Alain REY (dir.) (1994) : Le Nouveau Petit Robert, Paris, Dictionnaires Le Robert.

Riksmålsordboken (1977) : Oslo, Kunnskapsforlaget.

RYDNING, Antin Fougner (1991) : Qu'est-ce qu'une traduction acceptable en B ?, Thèse de doctorat d'État, Oslo, Université d'Oslo.

SELESKOVITCH, Danica et Marianne LEDERER (1989): Pédagogie raisonnée de l'interprétation, Paris, Didier Érudition.

St. meld. nr 25 (1992-1993) : Om mennesker og bioteknologi, Oslo, Sosialdepartementet.

Tanums store rettskrivningsordbok — bokmål (1979) : Oslo, Tanum — Norli.

VINAY, Jean-Paul et Jean DARBELNET (1977) : Stylistique comparée du français et de l'anglais, Paris, Didier. 